\title{
Amplification and squeezing of quantum noise with a tunable Josephson metamaterial
}

\author{
M. A. CASTELLANOS-BELTRAN ${ }^{1 *}$, K. D. IRWIN² , G. C. HILTON² , L. R. VALE ${ }^{2}$ AND K. W. LEHNERT ${ }^{1}$ \\ ${ }^{1}$ JILA, National Institute of Standards and Technology and the University of Colorado and Department of Physics, University of Colorado, Boulder, Colorado 80309, USA \\ ${ }^{2}$ National Institute of Standards and Technology, Boulder, Colorado 80305, USA \\ *e-mail: castellm@colorado.edu
}

It has recently become possible to encode the quantum state of superconducting qubits and the position of nanomechanical oscillators into the states of microwave fields ${ }^{1,2}$. However, to make an ideal measurement of the state of a qubit, or to detect the position of a mechanical oscillator with quantumlimited sensitivity, requires an amplifier that adds no noise. If an amplifier adds less than half a quantum of noise, it can also squeeze the quantum noise of the electromagnetic vacuum. Highly squeezed states of the vacuum can be used to generate entanglement or to realize back-action-evading measurements of position $^{3,4}$. Here we introduce a general-purpose parametric device, which operates in a frequency band between 4 and $8 \mathrm{GHz}$. It adds less than half a noise quantum, it amplifies quantum noise above the added noise of commercial amplifiers and it squeezes quantum fluctuations by $10 \mathrm{~dB}$.

With the emergence of quantum information processing with electrical circuits, there is a renewed interest in Josephson parametric devices ${ }^{5-9}$. Previous work with Josephson parametric amplifiers $^{10-14}$ demonstrated that they can act as phase-sensitive amplifiers that add less noise than the minimum noise for phaseinsensitive amplifiers ${ }^{15}$ and modestly squeeze vacuum noise. Earlier realizations of Josephson parametric amplifiers (JPAs) were capable of amplifying signals only in a narrow frequency range, were not operated with large enough gain to make the noise of the following, conventional amplifier negligible or added more than half a noise quantum owing to loss ${ }^{5}$. For related reasons, the degree of squeezing of the vacuum noise was never larger than $3 \mathrm{~dB}$. We have created a new type of parametric amplifier in which we have embedded a tunable, low-loss and nonlinear metamaterial in a microwave cavity. The tunability of the metamaterial enables us to adjust the amplified band between 4 and $8 \mathrm{GHz}$, and the cavity isolates the gain medium from low-frequency noise, providing the stability required to achieve high gains and large squeezing.

The time $(t)$ dependence of a single mode of a microwave field with angular frequency $\omega$ can be decomposed into two orthogonal components, referred to as quadratures,

$$
\hat{V}(t) \propto \hat{X}_{1} \cos \omega t+\hat{X}_{2} \sin \omega t,
$$

where $\hat{X}_{1}$ and $\hat{X}_{2}$ are conjugate quantum variables obeying the commutation relation $\left[\hat{X}_{1}, \hat{X}_{2}\right]=i / 2$. The proportionality constant depends on the details of the mode $e^{16-18}$. As a consequence of the commutation relation, the uncertainties in $\hat{X}_{1}$ and $\hat{X}_{2}$ are subject to the Heisenberg constraint $\Delta X_{1} \Delta X_{2} \geq 1 / 4$, where $\Delta X_{j}^{2}$ is the variance of the quadrature amplitude $\hat{X}_{j}$. A mode is 'squeezed' if for one of the quadratures $\Delta X_{j}<1 / 2$ (ref. 18). An amplifier that transforms both input quadratures by multiplying them by a gain $\sqrt{G}$ is a phase-insensitive amplifier and must add at least half a quantum of noise for the output signal to obey the commutation relation $^{15}$; if it adds exactly half a quantum of noise, it is quantum limited. On the other hand, an amplifier that transforms the quadratures of the input signal by different amounts is called a phase-sensitive amplifier and can add less than half a quantum of noise. An amplifier that multiplies one quadrature by $\sqrt{G}$ and multiplies the other quadrature by $1 / \sqrt{G}$ would have an output that satisfies the commutation relation automatically. It need not add any noise to the amplified output mode and, in this sense, it can be noiseless ${ }^{15,19}$. A degenerate parametric amplifier transforms the input signal in this way, amplifying one quadrature and deamplifying the other ${ }^{19}$.

Our realization of the parametric amplifier consists of a series array of 480 Josephson junctions, forming the centre conductor of a coplanar waveguide. Although the structure is composed of many discrete elements, it behaves as a continuous medium ${ }^{5}$, with a phase velocity and Kerr nonlinearity that emerges from the properties of the individual Josephson junctions; that is, it is a metamaterial. Each junction is split into two junctions in parallel, forming a superconducting quantum interference device (SQUID) and making the phase velocity of the metamaterial tunable with magnetic flux enclosed by the SQUID loops ${ }^{20}$ (Fig. 1a). In addition to being flux tunable, the phase velocity depends on the intensity of the fields propagating in this nonlinear metamaterial ${ }^{14}$. We have embedded this nonlinear medium in a half-wavelength cavity by terminating each end of the array with a capacitor. One capacitor is larger than the other, creating a strongly coupled port and a weakly coupled port.

We operate the parametric amplifier by injecting an intense pump tone into the weakly coupled port at a frequency close to the cavity's half-wave resonance. Through the intensity-dependent phase velocity, the pump causes the cavity's resonance frequency to vary at twice the pump's frequency, providing parametric gain. A signal incident on the strongly coupled port is reflected, and will be amplified if it is in phase with the pump but deamplified if it is $90^{\circ}$ out of phase with the pump. Signals leaving port 2 are 

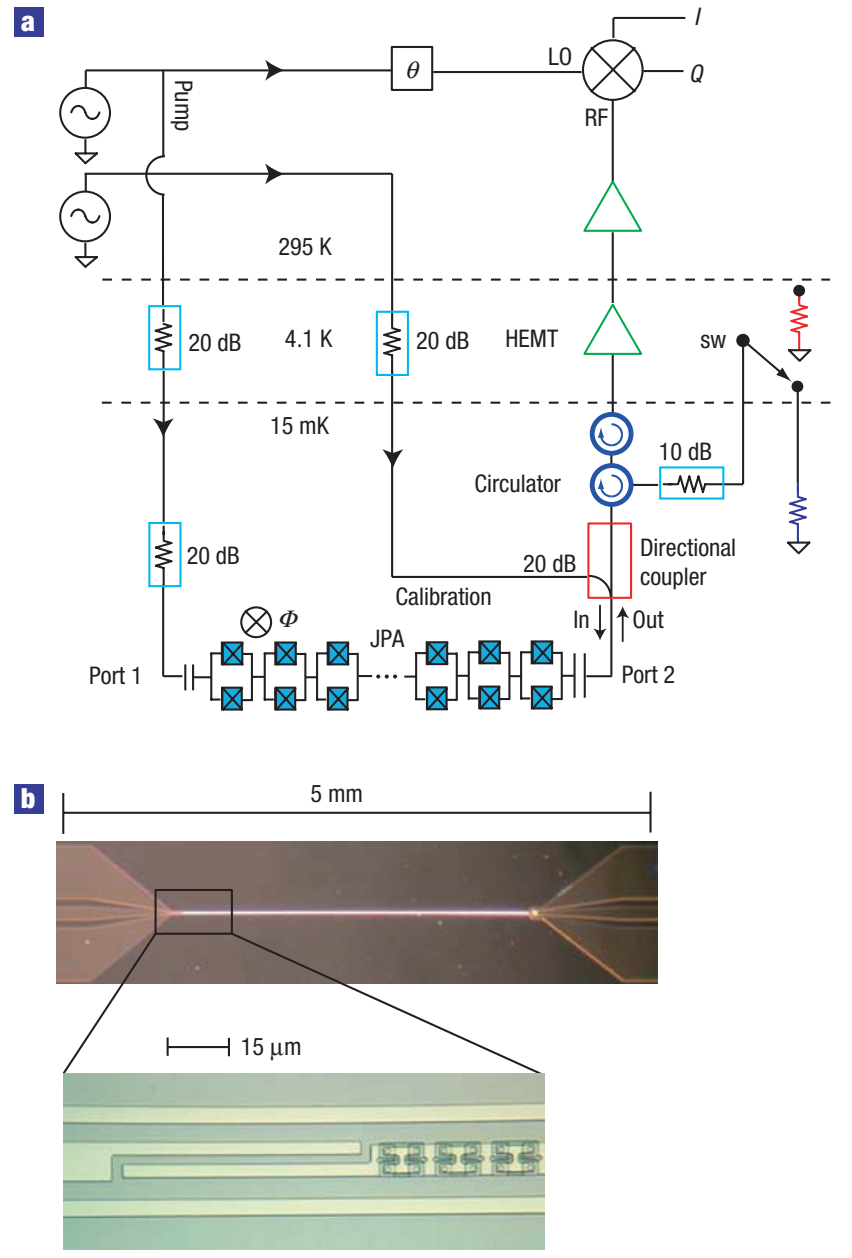

Figure 1 Measurement schematic diagram and device images. a, The JPA was measured in a cryostat held at $15 \mathrm{mK}$. Two microwave generators were used to study the JPA. One generator created the pump and local oscillator (LO) tones, whereas the second created a calibration tone. The pump tone was injected through port 1 of the JPA, and the calibration tone was incident on port 2 of the JPA through a $20 \mathrm{~dB}$ directional coupler. Signals emerging from port 2 passed through a circulator and were then amplified by a cryogenic high-electron-mobility transistor amplifier (HEMT) and a room-temperature amplifier before entering the radio-frequency (RF) port of a mixer. The noise incident on port 2 of the JPA, which came primarily from the isolated port of the circulator, could be switched (sw) to come from two different resistors held at different temperatures. $\mathbf{b}$, A picture of the full device (upper) and a magnified image of the weakly coupled port and a few SQUIDs (lower).

further amplified by a chain of commercial amplifiers. We resolve the components of the amplified signal that are in phase, $I$, and $90^{\circ}$ out of phase, $Q$, by mixing the signal with a phase reference called the local oscillator $(\mathrm{LO})$. Because the $\mathrm{LO}$ is derived from the same generator that produces the pump, $I$ and $Q$ are simply related to the quadrature components $\hat{X}_{1}$ and $\hat{X}_{2}$ of the microwave field exiting port 2. They are related by $I=\sqrt{A}\left(\hat{X}_{1} \cos \theta-\hat{X}_{2} \sin \theta+\xi_{I}(t)\right)$ and $Q=\sqrt{A}\left(\hat{X}_{1} \sin \theta+\hat{X}_{2} \cos \theta+\xi_{Q}(t)\right)$, where $A$ is the total power gain of the commercial amplifiers and mixer, $\theta$ is the phase between the pump and the LO, and $\xi_{I}$ and $\xi_{Q}$ are random variables. Both random variables have a power spectral density $N_{\mathrm{A}}$, where $N_{\mathrm{A}}$ is the noise number ${ }^{15}$ of the commercial amplifier chain expressed as noise added at the input of the HEMT (see Methods section).
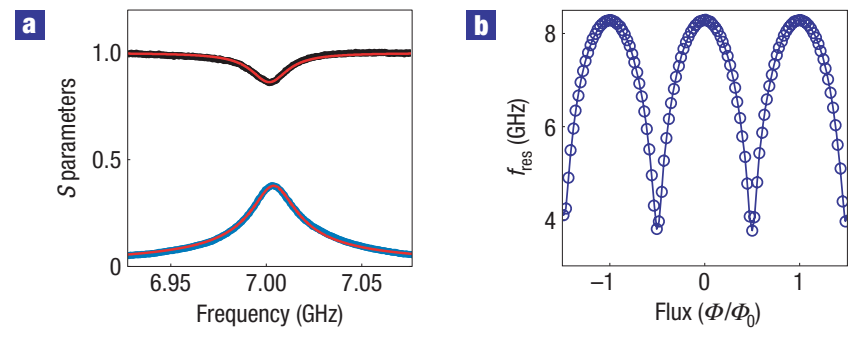

Figure 2 Linear response of the JPA. a, The fraction of microwave amplitude transmitted from port 1 to port $2\left|S_{21}\right|$ (blue) and the fraction reflected from port 2 $\left|S_{22}\right|$ (black) as functions of frequency at a particular value of applied magnetic flux. By fitting these data to a model of a two-port cavity (red lines), we extracted $f_{\text {res }}=7.0038 \mathrm{GHz}, \gamma_{\mathrm{c} 1}=2 \pi \times 437 \mathrm{kHz}, \gamma_{\mathrm{c} 2}=2 \pi \times 10.55 \mathrm{MHz}$ and $\gamma_{i}=2 \pi \times 341 \mathrm{kHz}$. $\mathbf{b}$, The measured value of $f_{\text {res }}$ as a function of applied magnetic flux $\Phi$ in units of the magnetic flux quantum $\Phi_{0}$. We used the obvious periodicity in these data to infer the magnetic flux enclosed by the SQUIDs.

Before operating the device as a parametric amplifier, we first characterized its linear behaviour. We measured the cavity's response by measuring both the reflectance of a signal on port 2 and the transmittance from port 1 to port 2 as functions of frequency (Fig. 2a). The powers used were low enough that the cavity's response was still linear. From this measurement we were able to extract the cavity's resonance frequency $f_{\text {res }}$ and the rates at which the cavity loses power through both ports, $\gamma_{\mathrm{cl}}$ and $\gamma_{\mathrm{c} 2}$, as well as through internal loss processes, $\gamma_{i}$ (Fig. 2a). When operating as a parametric amplifier, the centre of the amplified band will be close to $f_{\text {res }}$, and the unit-gain signal bandwidth will be approximately $\gamma_{\mathrm{c} 1}+\gamma_{\mathrm{c} 2}+\gamma_{i}=11.3 \mathrm{MHz}$ (refs 5,6); for example, if the JPA is operated with $\sqrt{G}=10$, the signal bandwidth is about $1.1 \mathrm{MHz}$. By applying magnetic flux we were able to move the centre of the amplified band over a large range of frequencies (Fig. 2b).

After characterizing the linear response of the device, we operated it as an amplifier. In previous work, we studied the dependence of the parametric gain on the pump power and pump frequency in our first realization of this metamaterial ${ }^{5}$, finding good agreement with the theory in ref. 6 . Here we study the noise performance of the JPA. We began by selecting $\theta=0$, so that the amplified quadrature appeared at the $I$ port of the mixer (Fig. 3a). To measure the noise added by the amplifiers, we monitored the noise at the $I$ port of the mixer while we switched between two sources of calibrated noise at the input of the JPA. The change in noise power at the output of the mixer enabled us to measure the noise $N_{\mathrm{JPA}}$ added by the JPA, the JPA gain $G$, the noise added by the commercial amplifier chain $N_{\mathrm{A}}$, the gain of the commercial amplifier chain $A$ and the total noise of the full amplifier chain $N_{\text {tot }}=N_{\text {IPA }}+N_{\mathrm{A}} / G$. The calibrated noise sources were derived from two resistors held at different temperatures (Fig. 1a). The noise emitted by both resistors was attenuated by a $10 \mathrm{~dB}$ attenuator held at a temperature $T_{\mathrm{A}}=15 \mathrm{mK}$ and then passed to the JPA through a microwave circulator, which separated the incident (input) and reflected (output) signals from the JPA. The power spectral density in units of noise quanta incident on the JPA through the circulator is

$$
N\left(T_{\mathrm{R}}\right)=\frac{1}{2}+\left(\frac{9}{10}\right) \frac{1}{\mathrm{e}^{\frac{\hbar \omega}{k_{B} T_{A}}}-1}+\left(\frac{1}{10}\right) \frac{1}{\mathrm{e}^{\frac{\hbar \omega}{k_{B} T_{\mathrm{R}}}}-1},
$$

where $T_{\mathrm{R}}=T_{\mathrm{c}}=15 \mathrm{mK}$ or $T_{\mathrm{R}}=T_{\mathrm{h}}=4.1 \mathrm{~K}$ depending on the position of the switch shown in Fig. 1a. The first term in 
a
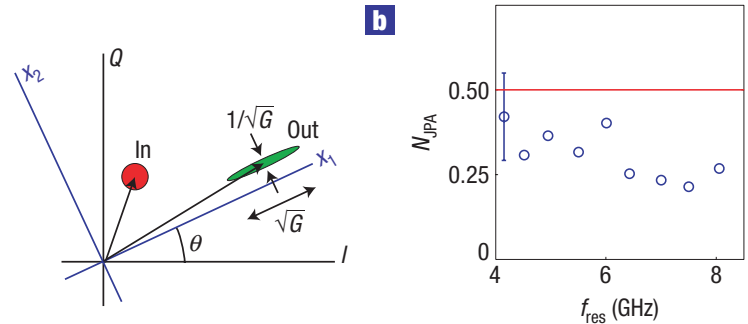

$\mathbf{G}$

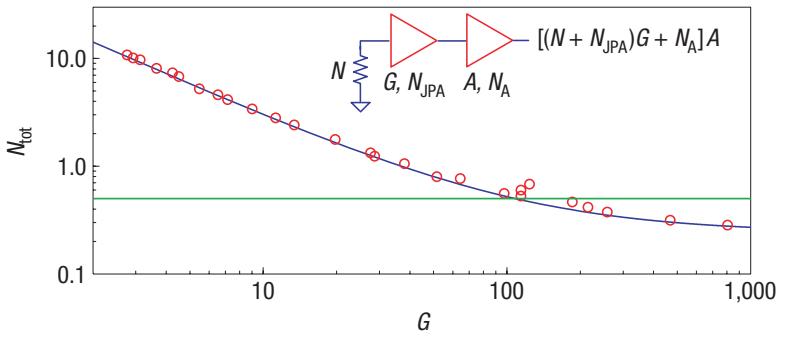

Figure 3 Added noise of the JPA and of the full measurement chain. a, The operation of an ideal degenerate parametric amplifier that transforms an input state (red circle) into an output state (green ellipse) by amplifying the $X_{1}$ component by $\sqrt{G}$ and squeezing the $X_{2}$ component by $1 / \sqrt{G}$. The mixer projects the output state into axes rotated by $\theta$. $\mathbf{b}$, We measured $N_{\text {JPA }}$ at nine different values of $f_{\text {res }}$ between 4 and $8 \mathrm{GHz}$ and observed that the added noise was less than half a quantum (red line). The error bar applies to all points; it represents the systematic uncertainty introduced primarily by the unknown loss of the directional coupler and switch when operated cryogenically. $\mathbf{c}, N_{\text {tot }}$ when $f_{\text {res }}=7.00 \mathrm{GHz}$ versus $G$ (points). At this frequency $N_{A}=26$ and is dominated by the HEMT. With increasing $G$, this noise becomes negligible compared with $N_{\mathrm{JPA}}=0.23$, where we expect $N_{\text {tot }}=N_{\text {JPA }}+N_{\mathrm{A}} / G$ (blue line). At the largest gains $N_{\text {tot }}<0.5$ (green line) and $N_{A} / G=0.04$. The inset diagram shows schematically the noise contributions of cascaded amplifiers. The JPA added $N_{\text {JPA }}$ to the noise power $N$ at its input. Both noises were multiplied by the JPA power gain $G$ before entering the input of the commercial amplifier chain. The cascaded amplifiers can be modelled as a single amplifier with added noise $N_{\text {tot }}$ and gain $A G$.

equation (1) is the quantum noise, whereas the second and third terms are the thermal noise, which is small compared with the quantum noise at $T_{\mathrm{R}}=T_{\mathrm{c}}$ (see Methods section). We measured the ratio $Y$ of the noise power at the output $I$ with the hot source incident on the JPA to the noise power with the cold source incident on the JPA. This measurement, known as a $Y$-factor measurement, enabled us to extract $N_{\text {tot }}=\left(N\left(T_{\mathrm{h}}\right)-Y N\left(T_{\mathrm{c}}\right)\right) /(Y-1)$ and the total gain $A G$. We then adjusted the cavity's resonance frequency about ten linewidths away from the LO frequency and turned off the pump. Because the JPA then acted as a passive mirror $\left(G=1, N_{\mathrm{IPA}}=0\right)$, we could make a $Y$-factor measurement on our commercial amplifier chain, finding $N_{\mathrm{A}}$ and $A$. From both measurements, we extracted $G$ and $N_{\text {IPA }}$ (Fig. 3). As observed in Fig. 3b, our JPA added less than half a quantum of noise over the $4-8 \mathrm{GHz}$ band where it operated. In addition, it could be operated at large enough gain to amplify the quantum noise above the commercial amplifier's noise (Fig. 3c).

To show that the JPA could squeeze quantum noise, we examined the squeezed quadrature $X_{2}$ when the incident noise is mostly quantum noise $\left(T_{\mathrm{R}}=T_{\mathrm{c}}\right)$. When $\theta=0, X_{2}$ appears at the $Q$ port. The noise at the $Q$ port referred to the input of the HEMT is composed of the added noise of the commercial amplifiers $\left(N_{\mathrm{A}} \approx 26\right)$ and noise coming from port $2,2 \Delta X_{Q}^{2}$, where $\Delta X_{Q}^{2}=\Delta X_{1}^{2} \sin ^{2} \theta+\Delta X_{2}^{2} \cos ^{2} \theta$. When $\theta=0$, we observed
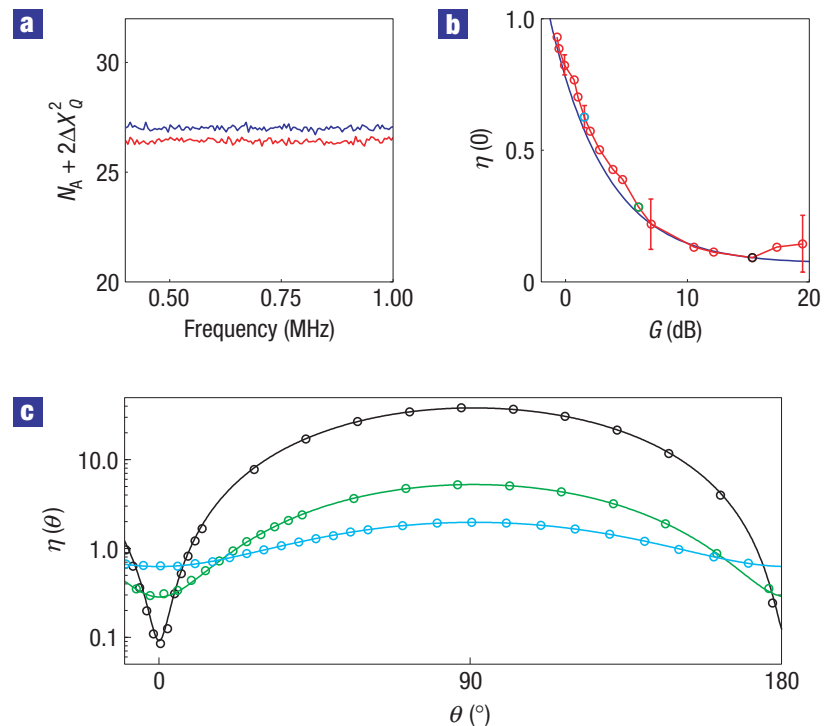

Figure 4 Demonstration of squeezing. a, Power spectral density versus frequency at the $Q$ output of the mixer referred to the input of the commercial amplifier chain in units of quanta, measured with the pump on (red) and the pump off (blue) for $\theta=0, f_{\text {res }}=7 \mathrm{GHz}$ and $G=15.3 \mathrm{~dB}$. $\mathbf{b}$, The squeezing $\eta(0)$ as a function of $G$ (points) and the predicted squeezing from ref. 6 (line). The error bars represent the same systematic error as in Fig. 3b. This uncertainty varies smoothly between the error bars shown at selected data points. c, The quantity $\eta(\theta)$, defined in the text, as a function of $\theta$ for three different gains (points), $G=34$ (black), $G=4$ (green) and $G=1.4$ (cyan), and the expected $\theta$ dependencies (lines) ${ }^{6,12}$.

a reduction in $\Delta X_{Q}$ when the pump was turned on, demonstrating that the JPA had squeezed quantum noise (Fig. 4a). As we increased the JPA gain, we observed an increase in the noise squeezing up to $G=16 \mathrm{~dB}$ (Fig. 4b). For $G<16 \mathrm{~dB}$ the squeezing that we observed was consistent with the expected behaviour from a model of parametric amplification that includes loss in the JPA but no other imperfections ${ }^{6}$. However, for $G>16 \mathrm{~dB}$ we observed less than the predicted squeezing, probably owing to the instability in the phase acquired by the pump as it passed through the cryostat in the several minutes required to complete one measurement. Finally, to illustrate the JPA's unequal effect on the noise of the two quadratures, we defined a

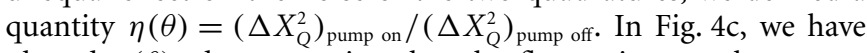
plotted $\eta(\theta)$, demonstrating that the fluctuations at the output of the JPA are indeed squeezed along the axis defined by the pump (Fig. 3a).

The measurement of the squeezed state would have proceeded much more rapidly if we had used a second JPA instead of a HEMT amplifier, which adds 26 noise quanta. For most measurements, the low added noise of the JPA is helpful, but for some measurements it is crucial. For example, to fully characterize the quantum state of non-Gaussian states, such as the Fock states generated by superconducting qubits ${ }^{2}$, a nearly noiseless amplifier is a necessity ${ }^{21}$. In addition to characterizing non-Gaussian states of microwave fields, the JPA is also well suited to amplifying the signal generated by a nanomechanical beam moving in a microwave cavity $^{1}$, allowing a quantum-limited measurement of position. The tunability of the amplifier demonstrated here also makes it well suited to use with the increasingly popular frequencydivision-multiplexed microwave circuits ${ }^{1,2,23}$ when simultaneous measurement of all frequency channels is not required. 


\section{METHODS}

Devices were fabricated at NIST Boulder using a standard $\mathrm{Nb}-\mathrm{AlO}_{x}-\mathrm{Nb}$ trilayer process $^{24}$, modified by eliminating the shunt resistor layer and minimizing deposited oxides ${ }^{23}$. The device studied here was coprocessed with ten other devices used for d.c. characterization. These d.c. measurements indicate an average critical current $I_{\mathrm{c}}$ per SQUID in the JPA of $31 \mu \mathrm{A}$, which was close to the designed value of $30 \mu \mathrm{A}$. The transmission lines from which the half-wavelength cavities were built were designed to have a capacitance per unit length of $C_{\mathrm{l}}=0.15 \mathrm{nF} \mathrm{m}^{-1}$ and a geometrical inductance per unit length of $L_{1}=0.49 \mu \mathrm{H} \mathrm{m}^{-1}$. For $I_{\mathrm{c}}=31 \mu \mathrm{A}$, the Josephson inductance per SQUID is $L_{\mathrm{J}}=10.6 \mathrm{pH}$. These values predict a half-wave resonance frequency of $8.23 \mathrm{GHz}$ when no flux is applied, which is within $3 \%$ of the observed value. Measurements were made in a magnetically shielded dilution refrigerator. To avoid unknown loss and noise in the cables carrying the calibration noise signals, superconducting niobium coaxial cables were used to carry these signals between the $15 \mathrm{mK}$ region of the refrigerator and the $4 \mathrm{~K}$ region. A calibrated ruthenium oxide thermometer measured the refrigerator's base temperature and the temperature of the helium bath, to which the $4 \mathrm{~K}$ resistor and microwave switch were thermally anchored. A calibration tone (Fig. 1a) was used to verify the gain inferred from the $Y$-factor measurements, to ensure that the noise reduction arose from genuine squeezing and not saturation of the amplifiers, and to measure $S_{22}$ in the network analysis. The complete expression for the noise incident on the JPA includes contributions from the thermal noise introduced with the calibration tone and the insertion loss of the directional coupler and the switch. The unknown insertion loss at cryogenic temperatures of the directional coupler and of the microwave switch are the dominant uncertainties in the added noise and squeezing measurements. The extreme values represented by the error bars were derived by assuming first that there was no insertion loss, and then that the insertion loss had not changed between room temperature and the operating temperature. Noise at the $I$ or the $Q$ ports was expressed as the double-side-band power spectral density referred to the input of the relevant amplifier. Dividing the spectral density by $h f_{\mathrm{LO}}$ yields the noise in dimensionless units of noise quanta, where $f_{\mathrm{LO}}$ is the local oscillator frequency; for example, the thermal noise of a resistor at temperature $T \gg h f_{\mathrm{LO}} / k_{\mathrm{B}}$ measured at the $I$ port would have a noise $N_{T}=k_{\mathrm{B}} T / h f_{\mathrm{LO}}$. Noise densities were measured by sampling the mixer outputs at $10 \mathrm{MHz}$ with a $5 \mathrm{MHz}$ antialias filter for a period of time of $400 \mu \mathrm{s}$. We calculated the power spectral density and repeated and averaged the measurement 25,000 times. The added noise that we quote is an average of the power spectral density over the frequency window $100 \mathrm{kHz}$ to $4 \mathrm{MHz}$.

Received 13 May 2008; accepted 1 September 2008; published 5 0ctober 2008.

References

1. Regal, C. A., Teufel, J. D. \& Lehnert, K. W. Measuring nanomechanical motion with a microwave cavity interferometer. Nature Phys. 4, 555-560 (2008).
2. Houck, A. A. et al. Generating single microwave photons in a circuit. Nature 449, 328-331 (2007)

3. Braunstein, S. L. \& van Loock, P. Quantum information with continuous variables. Rev. Mod. Phys. 77, 513-577 (2005).

4. Caves, C. M., Thorne, K. S., Drever, R. W. P., Sandberg, V. D. \& Zimmermann, M. On the measurement of a weak classical force coupled to a quantum-mechanical oscillator. I. Issues of principle. Rev. Mod. Phys. 52, 341-392 (1980).

5. Castellanos-Beltran, M. A. \& Lehnert, K. W. Widely tunable parametric amplifier based on a superconducting quantum interference device array resonator. Appl. Phys. Lett. 91, 083509 (2007).

6. Yurke, B. \& Buks, E. Performance of cavity-parametric amplifiers, employing Kerr nonlinearities, in the presence of two-photon loss. J. Lightw. Technol. 24, 5054-5066 (2006).

7. Tholén, E. A. et al. Nonlinearities and parametric amplification in superconducting coplanar waveguide resonators. Appl. Phys. Lett. 90, 253509 (2007).

8. Siddiqi, I. et al. Rf-driven Josephson bifurcation amplifier for quantum measurement. Phys. Rev. Lett. 93, 207002 (2004).

9. Zagoskin, A., Il'ichev, E., McCutcheon, M. W., Young, J. \& Nori, F. Generation of squeezed states of microwave radiation in a superconducting resonant circuit. Preprint at $<$ http://arxiv.org/abs/0804.4186vl > (2008).

10. Yurke, B. Squeezed-state generation using a Josephson parametric amplifier. J. Opt. Soc. Am. B 4, 1551-1557 (1987).

11. Yurke, B. et al. Observation of 4.2-K equilibrium-noise squeezing via a Josephson-parametric amplifier. Phys. Rev. Lett. 60, 764-767 (1988).

12. Yurke, B. et al. Observation of parametric amplification and deamplification in a Josephson parametric amplifier. Phys. Rev. A 39, 2519-2533 (1989).

13. Movshovich, R. et al. Observation of zero-point noise squeezing via a Josephson-parametric amplifier. Phys. Rev. Lett. 65, 1419-1422 (1990).

14. Yurke, B., Roukes, M. L., Movshovich, R. \& Pargellis, A. N. A low-noise series-array Josephson junction parametric amplifier. Appl. Phys. Lett. 69, 3078-3080 (1996).

15. Caves, C. M. Quantum limits on noise in linear amplifiers. Phys. Rev. D 26, 1817-1839 (1982).

16. Yurke, B. \& Denker, J. S. Quantum network theory. Phys. Rev. A 29, 1419-1437 (1984).

17. Louisell, W. H. Radiation and Noise in Quantum Electronics Ch. 4, 137-174 (McGraw-Hill Book Company, 1964).

18. Gerry, C. C. \& Knight, P. L. Introductory Quantum Optics (Cambridge Univ. Press, 2006).

19. Takahasi, H. in Advances in Communication Systems (ed. Balakrishnan, A. V.) Ch. 6, 227-310 (Academic, 1965).

20. Haviland, D. B. \& Delsing, P. Cooper-pair charge solitons: The electrodynamics of localized charge in a superconductor. Phys. Rev. B 54, R6857-R6860 (1996).

21. Yurke, B. \& Stoler, D. Generating quantum mechanical superpositions of macroscopically distinguishable states via amplitude dispersion. Phys. Rev. Lett. 57, 13-16 (1986).

22. Day, P. K., LeDuc, H. G., Mazin, B. A., Vayonakis, A. \& Zmuidzinas, J. A broadband superconducting detector suitable for use in large arrays. Nature 425, 817-821 (2003).

23. Mates, J. A. B., Hilton, G. C., Irwin, K. D., Vale, L. R. \& Lehnert, K. W. Demonstration of a multiplexer of dissipationless superconducting quantum interference devices. Appl. Phys. Lett. 92 023514 (2008).

24. Sauvageau, J. et al. Superconducting integrated circuit fabrication with low temperature ECR-based PECVD $\mathrm{SiO}_{2}$ dielectric films. IEEE Trans. Appl. Supercond. 5, 2303-2309 (1995).

\section{Acknowledgements}

The authors acknowledge funding from the National Institute of Standards and Technology (NIST), from the National Science Foundation and from a NIST-University of Colorado seed grant. We thank S. M. Girvin, J. D. Teufel, C. A. Regal, N. E. Flowers-Jacobs, J. K. Thompson and M. Holland for conversations and technical assistance. K.W.L. is a member of NIST's Quantum Physics Division.

\section{Author contributions}

Project planning was done by M.A.C., K.D.I., G.C.H. and K.W.L. Experimental work was carried out by M.A.C. and K.W.L. Data analysis was carried out by M.A.C. and K.W.L. Samples were fabricated by G.C.H. and L.R.V.

\section{Author information}

Reprints and permissions information is available online at http://npg.nature.com/reprintsandpermissions. Correspondence and requests for materials should be addressed to M.A.C. 